\title{
CEN 34 - high-mass YSO in M 17 or background post-AGB star? ${ }^{\star}$
}

\author{
Zhiwei Chen ${ }^{1,2,3}$, Dieter E. A. Nürnberger ${ }^{2}$, Rolf Chini ${ }^{2,4}$, Yao Liu ${ }^{1}$, Min Fang ${ }^{1}$, and Zhibo Jiang ${ }^{1}$ \\ 1 Purple Mountain Observatory, Chinese Academy of Sciences, 2 West Beijing Road, 210008 Nanjing, PR China \\ e-mail: zwchen@pmo.ac.cn \\ 2 Astronomisches Institut, Ruhr-Universität Bochum, Universitätsstrasse 150, 44801 Bochum, Germany \\ 3 University of Chinese Academy of Sciences, 100039 Beijing, PR China \\ ${ }^{4}$ Instituto de Astronomía, Universidad Católica del Norte, Avenida Angamos 0610, Casilla 1280 Antofagasta, Chile
}

Received 14 April 2013 / Accepted 10 July 2013

\begin{abstract}
We investigate the proposed high-mass young stellar object (YSO) candidate CEN 34, thought to be associated with the star-forming region M 17. Its optical to near-infrared $(550-2500 \mathrm{~nm})$ spectrum reveals several photospheric absorption features, such as $\mathrm{H} \alpha$, the Ca II triplet, and the CO bandhead, but lacks emission lines. The spectral features in the range 8375-8770 A are used to constrain an effective temperature $T_{\text {eff }}=5250 \pm 250 \mathrm{~K}$ (early- $/ \mathrm{mid}-\mathrm{G}$ ) and a $\log g=2.0 \pm 0.3$ (supergiant). The spectral energy distribution (SED) displays a faint infrared excess that resembles that of a high-mass YSO or an evolved star of intermediate mass. Moreover, the observed temperature and surface gravity are identical for high-mass YSOs and evolved stars. The radial velocity of CEN 34 relative to the local standard of rest $\left(V_{\mathrm{LSR}}\right)$ as obtained from various photospheric lines is of the order of $-60 \mathrm{~km} \mathrm{~s}^{-1}$ and thus distinct from the $+25 \mathrm{~km} \mathrm{~s}^{-1}$ found for several OB stars in the cluster and for the associated molecular cloud. The SED modeling yields $\sim 10^{-4} M_{\odot}$ of circumstellar material, which contributes only a tiny fraction to the total visual extinction (11 mag). The distance of CEN 34 is between $2.0 \mathrm{kpc}$ and $4.5 \mathrm{kpc}$. In the case of a YSO, a dynamical ejection process is proposed to explain the $V_{\mathrm{LSR}}$ difference between CEN 34 and M 17. Additionally, to match the temperature and luminosity, we speculate that CEN 34 had accumulated the bulk of its mass with an accretion rate $>4 \times 10^{-3} M_{\odot} /$ yr over a very short time span $\left(\sim 10^{3} \mathrm{yrs}\right)$, and it is currently undergoing a phase of gravitational contraction without any further mass gain. However, all the aforementioned characteristics of CEN 34 are compatible with an evolved star of 5-7 $M_{\odot}$ and an age of 50-100 Myr, so it is most likely a background post-AGB star with a distance between $2.0 \mathrm{kpc}$ and $4.5 \mathrm{kpc}$. We consider the latter classification as the more likely interpretation. Further discrimination of the two possible scenarios should come from the stricter confinement of CEN 34's distance.
\end{abstract}

Key words. stars: late-type - stars: AGB and post-AGB - stars: formation - stars: pre-main sequence

\section{Introduction}

Before reaching the main sequence, young stars accumulate the bulk of their mass during the so-called pre-main sequence (PMS) evolutionary phase. This phase can last as long as a few Myr for low- and intermediate-mass stars $\left(<8 M_{\odot}\right)$, while it can be as short as $\sim 10^{4}-10^{5} \mathrm{yr}$ for high-mass stars $\left(>8 M_{\odot}\right.$; Zinnecker \& Yorke 2007). During the majority of their PMS phase, young stars are surrounded and obscured by significant amounts of molecular gas and dust; the younger the forming stars, the more deeply embedded they are in their natal environments, and thus, the more difficult their detection and characterization through observations at optical and near-infrared (NIR) wavelength (e.g., Nielbock et al. 2007). The detection of high-mass young stellar objects (YSOs) is rare, because these objects are deeply embedded, but equally hampering is the extremely short PMS evolutionary phase, which makes it statistically more difficult to find them. Furthermore, the search for and the identification of highmass YSOs is hampered by confusion with much more evolved objects, showing similar or even identical observational signatures. For instance, if infrared (IR) excess is considered as the sole criterion for source selection, high-mass YSO candidates and post-asymptotic giant branch (post-AGB) stars might be

\footnotetext{
* Based on observations performed with the ESO Very large Telescope on Cerro Paranal, Chile, as part of the X-shooter Science Verification program 60.A-9402(A).
}

easily mixed up, although they are totally distinct in evolutionary phase. In both cases the observed IR excess originates in absorption of stellar photons by circumstellar dust and subsequent re-emission as thermal radiation at longer wavelengths. Alternatively, both the forming high-mass YSOs and the evolved stars have larger stellar radii and correspondingly cooler effective temperatures than the main sequence stars. This also prevents distinguishing high-mass YSOs from evolved stars solely from a spectroscopic point-of-view.

As one of the brightest H II regions in the Galaxy, M 17 shows evidence of multiple epochs of massive star formation (Jiang et al. 2002; Povich et al. 2009; Chen et al. 2012). It features two prominent bars, the northern $\mathrm{H}$ II bar (N-bar) and the southwestern H II bar (S-bar), which are ionized by a couple of OB-type stars located in the center of the H II region (Chen et al. 2012). A number of intermediate- and highmass YSOs have been discovered in the S-bar (Chini et al. 2004; Nürnberger et al. 2007; Nielbock et al. 2007; Ochsendorf et al. 2011). In addition, the S-bar harbors several high-mass YSO candidates, which display rising spectral energy distributions (SEDs) towards IR wavelengths but still await confirmation of their preliminary classification (Nielbock et al. 2001). Among these high-mass YSO candidates, CEN 34 (also known as B 358) has its own confusing peculiarities. It is very bright in the near IR $(K(2 \mathrm{MASS})=7.88 \mathrm{mag})$ and rather faint at optical wavelengths $(B \sim 22.8 \mathrm{mag}, V \sim 18.8 \mathrm{mag})$, which is indicative 


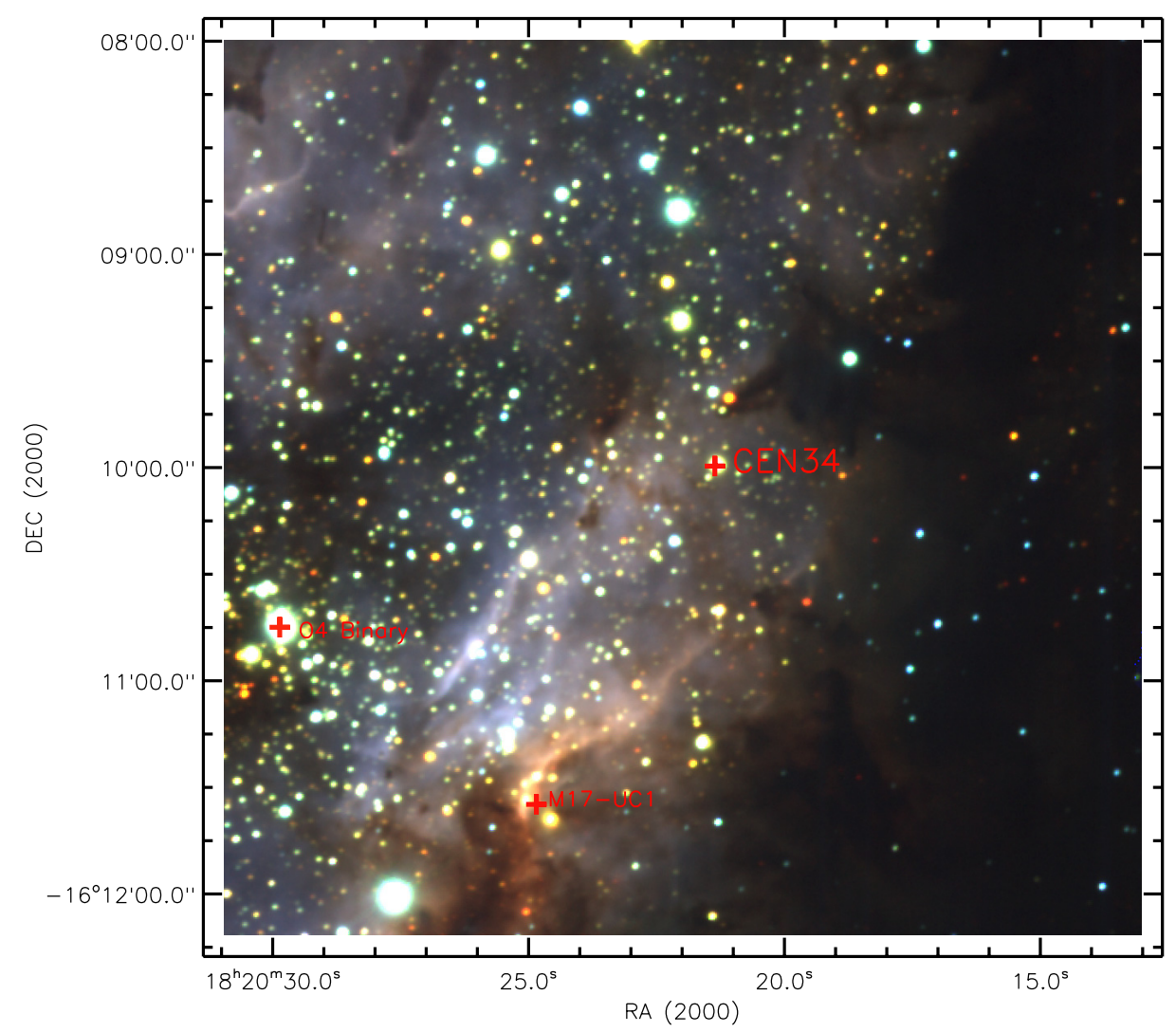

Fig. 1. The projection of CEN 34 (red cross) on the sky overlaid on the $J H K_{\mathrm{s}}$ pseudocolor image of M 17 taken by the InfraRed Survey Facility (IRSF) telescope. The ionizing sources of M 17-the O4-type binary, and the hypercompact $\mathrm{H}$ II region-M 17-UC1 are also marked. of high extinction. Its $K$-band spectrum shows the CO bandheads longwards of $2.29 \mu \mathrm{m}$ in absorption (Hanson et al. 1997; Hoffmeister et al. 2006), reminiscent of late-type Class II/III YSOs (Casali \& Eiroa 1996; Hoffmeister et al. 2006). However, the same feature is typical of late-type stars, as well as of evolved red stars (e.g., Wallace \& Hinkle 1997). Therefore, the true nature of CEN 34 is unclear.

To constrain the effective temperature and luminosity class of CEN 34, which is crucial to better understanding its nature, we performed new spectroscopic observations with high sensitivity and large wavelength coverage (from the optical to the NIR), using the medium-resolution spectrograph X-shooter on ESO's Very Large Telescope (VLT). In this paper we report on our findings with respect to CEN 34's spectral classification, its radial velocity in comparison to that of other cluster members, its extinction and its most likely distance.

\section{Observations and data reduction}

The VLT/X-shooter observations of CEN $34\left(18^{\mathrm{h}} 20^{\mathrm{m}} 21^{\mathrm{s}} .36\right.$, $-16^{\circ} 09^{\prime} 59^{\prime}$. 6 J2000) were performed on August 12, 2009 during the first science verification run of X-shooter, which is the first of the second-generation instruments at the VLT (D'Odorico et al. 2006; Vernet et al. 2011). The average optical seeing and airmass during the observations are 0.'59 and 1.376, respectively. All observations were acquired by nodding the star on the slit using an ABBA sequence, allowing for proper sky subtraction and bad pixel removal. On each nodding pointing, the integration time is $685 \mathrm{~s}$ for the UVB arm (300-600 nm), $570 \mathrm{~s}$ for the VIS arm $(550-1000 \mathrm{~nm})$, and $33 \mathrm{~s}$ for the NIR arm $(1000-2500 \mathrm{~nm})$. In total, the on-source integration time is $45 \mathrm{~min}$ for the UVB arm, 38 min for the VIS arm, and 2.2 min for the NIR arm. The data taken in the UVB arm were not satisfying because the faintness of CEN 34 provided an insufficient signal-to-noise $(\mathrm{S} / \mathrm{N})$.
For the VIS and the NIR arms, a 0.9 slit width was used to guarantee $R=8800(\Delta \lambda=0.09 \mathrm{~nm})$ and $R=5600(\Delta \lambda=0.3 \mathrm{~nm})$, respectively. The $\mathrm{S} / \mathrm{N}$ is $\sim 30$ in the VIS arm and $\sim 20$ in the NIR arm. The standard procedures of data reduction were applied using the X-shooter pipeline version 1.3.7 (Goldoni 2011), including bias-, dark-, and flat-field correction, wavelength calibration, order tracing, and flux calibration. Regarding the wavelength calibration, the transformation from pixel space to $(\lambda, \mathrm{s})$ space was performed by analyzing a nine-pinhole Th-Ar lamp frame with a list of lines and a table containing the expected positions of the lines in the frame. The accuracy of wavelength calibration using the pipeline is better than $\sim 2 \mathrm{~km} \mathrm{~s}^{-1}$ over the entire wavelength range of X-shooter. The standard star EG 274 (a white dwarf) was used to calibrate the fluxes for the spectra of the three individual arms. Meanwhile, the telluric absorption correction was conducted for the spectrum of the NIR arm by using the telluric standard stars HD 100858.

\section{Results}

The relative location of CEN 34 with respect to the $\mathrm{H}$ II region is shown in the finding chart (see Fig. 1). The most massive stars, the O 4-type binary (Hoffmeister et al. 2008), are located on the left edge of the finding chart, meanwhile the giant molecular cloud M 17-SW is located on the right edge.

The flux-calibrated spectrum of CEN 34 from the visual to the NIR is shown in Fig. 2. The optical and NIR parts of the spectrum are quite noisy due to the faintness at optical wavelengths and the short exposure time at NIR wavelengths, respectively. Only absorption lines are detected, such as $\mathrm{H} \alpha$, Ca II triplet, and $\mathrm{CO}$ bandheads in the $K$-band. The analysis based on the $\mathrm{Ca}$ II triplet and CO bandheads are presented in the following sections. 


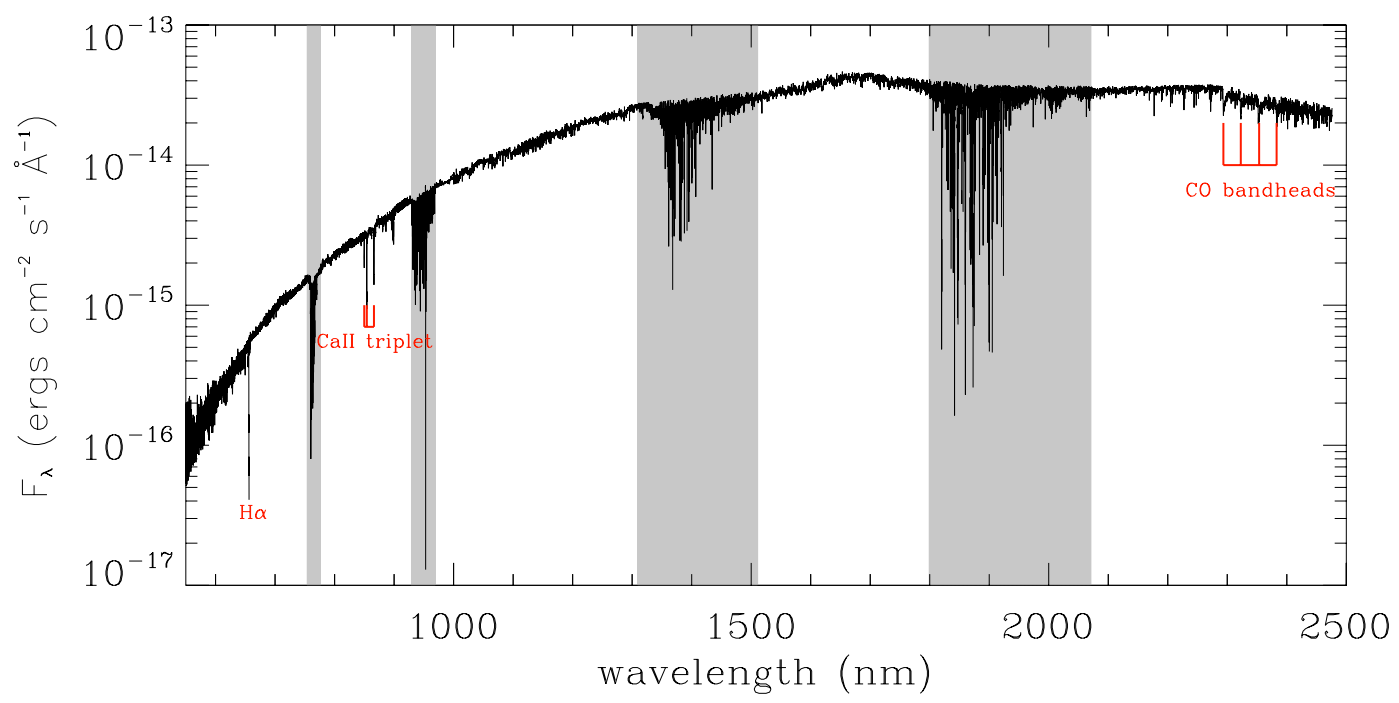

Fig. 2. X-shooter spectrum of CEN 34, covering the visible and near-IR wavelength range. Prominent photospheric absorption lines are marked. Gray shaded regions mark the major telluric absorption features.

Table 1. Line parameters.

\begin{tabular}{lcc}
\hline \hline $\begin{array}{l}\lambda_{\text {obs }}\left(\lambda_{0}\right) \\
{[\AA]}\end{array}$ & Ion & $\begin{array}{c}\text { Intensity } \\
\text { in absorption }\end{array}$ \\
\hline 8437 & Ti I & 0.10 \\
8446 & O I & 0.15 \\
8468 & Fe I & 0.10 \\
$8496.6(8498.02)$ & Ca II & 0.43 \\
8515 & Fe I & 0.09 \\
$8540.3(8542.09)$ & Ca II & 0.72 \\
8598 & H I & 0.09 \\
8621 & Fe I & 0.15 \\
$8660.5(8662.14)$ & Ca II & 0.61 \\
8689 & Fe I & 0.18 \\
\hline
\end{tabular}

Notes. The intensities of the lines are normalized line strengths with respect to the continuum. For the Ca II triplet, the references of rest wavelength in air (within the bracket; Reader et al. 1980) are given for the purpose of calculating the $V_{\mathrm{LSR}}$ of CEN 34. The weaker lines have S/Ns between 5 and 9 were not used for velocity determinations, so their rest frame wavelengths are not listed.

\subsection{Spectral type and luminosity class}

Our spectral classification of CEN 34 relies on the wavelength range $8375-8770 \AA$, which avoids strong telluric absorption. It also offers a rich combination of lines relevant for astrophysical diagnostics and spectral classification (Gray \& Corbally 2009). First, neutral $\mathrm{Fe}$ and $\mathrm{Ti}$ lines appear from late-F stars onwards, and grow gradually in intensity with decreasing temperature until late-K stars. Second, the Paschen series (e.g., Paschen 14 line $8598 \AA$, hereafter P14) disappear for main sequence stars later than G0, but they can still be seen for mid-G supergiants due to the luminosity effect (Munari \& Tomasella 1999). Third, neutral Ti and Fe lines, the Ca II triplet (8498 $\AA$, $8542 \AA, 8662 \AA$ ), as well as the lines of the Paschen series can provide luminosity discrimination by comparing their absolute strengths.

Figure 3 shows the wavelength range 8375-8770 $\AA$ of the $\mathrm{X}$-shooter spectrum of CEN 34 in comparison to the spectra of several reference stars of luminosity classes I (supergiants);

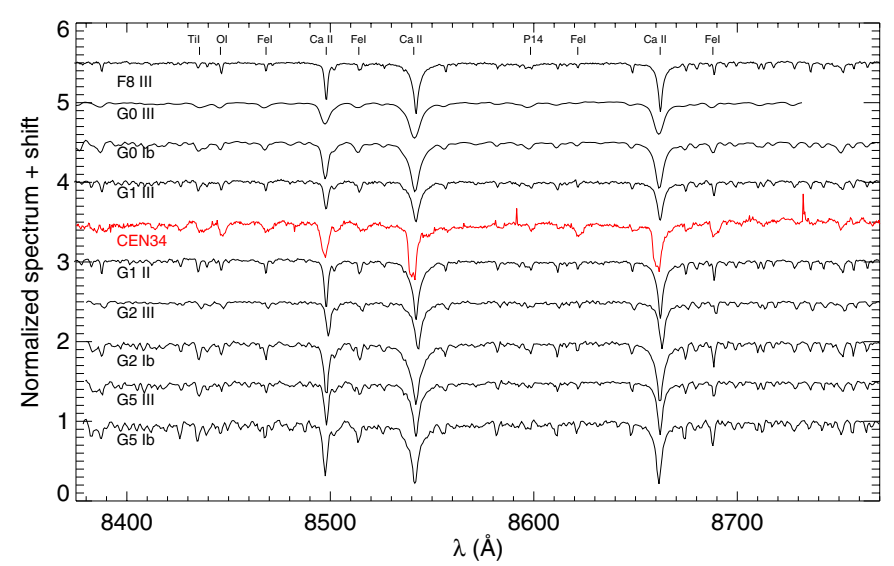

Fig. 3. X-shooter spectrum of CEN 34 (emphasized in red) in the wavelength region $8375-8770 \AA$. The two features apparently seen in emission in the CEN 34 spectrum at wavelengths $\sim 8590 \AA$ and $\sim 8730 \AA$ are artifacts with unnatural line widths. For comparison, the spectra of several reference stars are displayed; they are adopted from the literature as follows: F8 III (HIP 74975), G1 III (HIP 117503), and G1 II (HIP 47908) from Valdes et al. (2004); G0 Ib (HIP 96481) and G0 III (HIP 5454) from Andrillat et al. (1995); G2 Ib (HIP 109074), G2 III (HIP 86731), G5 III (HIP 100524), and G5 Ib (HIP 107348) from Carquillat et al. (1997). All reference spectra have a resolution around $1 \AA$, very close to that of our CEN 34 spectrum. All spectra are normalized and are displayed by applying a corresponding shift along the Y-axis for better visual comparison.

II (bright giants); and III (normal giants). Prominent absorption lines - like the Ca II triplet, neutral metal lines (Fe I, Ti I, and $\mathrm{OI}$ ), and the P14 line - are marked, and their derived parameters listed in Table 1. The appearance of Fe I, Ti I, and Paschen lines in the spectrum of CEN 34 indicates a rough temperature class of late-F to mid-G. The absolute strengths of these absorption lines are greater than those for giants, suggesting a luminosity class II or I. In addition, Ti I and Paschen lines stronger than in the spectrum of the F8 III reference star can be due to the brighter luminosity class (II or I) and/or later temperature class. 

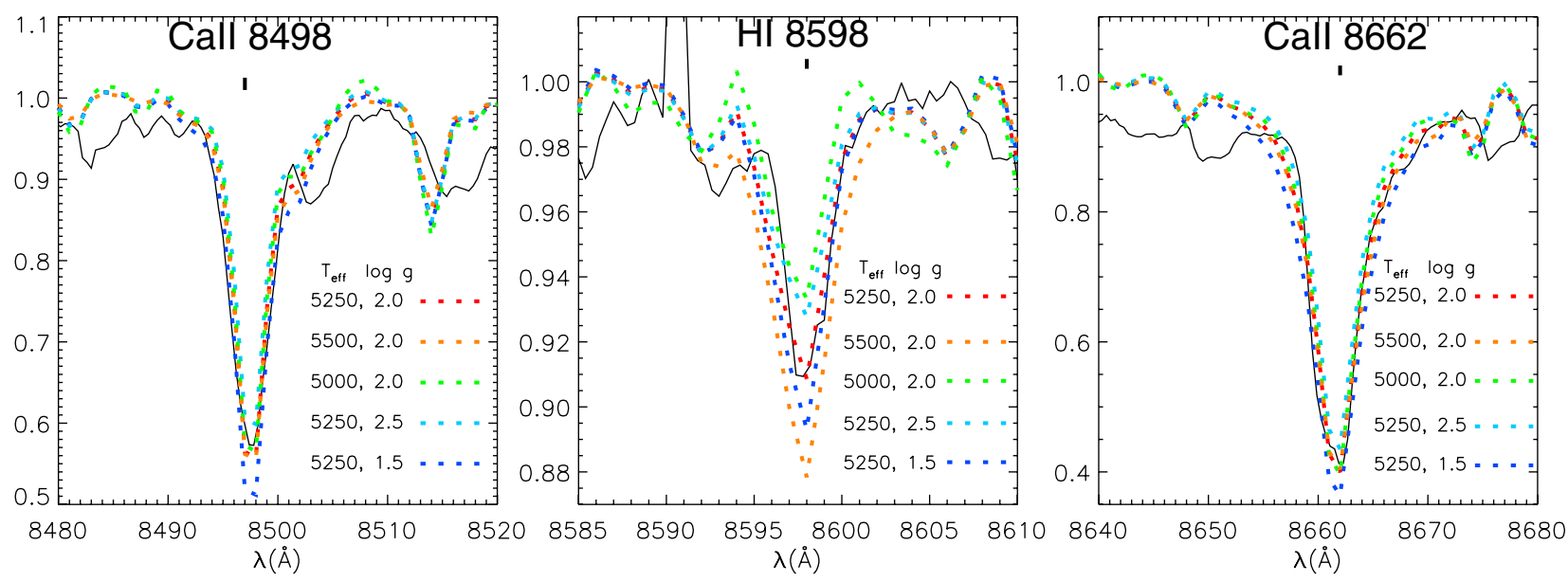

Fig. 4. CEN 34's X-shooter spectrum (black solid) around the Ca II $8498 \AA$ A, P14, and Ca II $8662 \AA$ lines. The best fitting grid (red) and other nearby grids are shown as dashed lines. The synthetic spectral library is based on the Kurucz codes, and has an uniform dispersion of $1 \AA$ Ápix (for more details see Munari et al. 2005).

\subsection{Effective temperature and surface gravity}

Effective temperature and gravity of CEN 34 are further constrained by comparing the observed spectrum to the library of synthetic spectra published by Munari et al. (2005) and assuming solar metallicity. The metallicity-dependent Ca II triplet is used as the primary discriminator because of its line strength and best $\mathrm{S} / \mathrm{N}$ ratio. In addition, to rule out any metallicity effect, the metallicity independent P14 line is used as secondary discriminator.

We construct a grid of synthetic spectra by covering the parameter space $5000 \mathrm{~K} \leqslant T_{\text {eff }} \leqslant 6000 \mathrm{~K}, \Delta T=250 \mathrm{~K}$, $0.0 \leqslant \log g \leqslant 3.0, \Delta \log g=0.5$. On the basis of the spectral type of CEN 34, we suggest a rotation velocity of $10 \mathrm{~km} \mathrm{~s}^{-1}$ (the typical value for early-G supergiants; De Medeiros et al. 2002) during the fitting procedure. The best fitting synthetic spectrum should first match the Ca II triplet and then match the P14 line; fitting results are checked by visual inspection alone. Figure 4 shows the best fitting grid and other nearby grids. Among the five different grids, we notice that the line strengths of the Ca II lines are very sensitive to $\log g$, while not varying much with $T_{\text {eff }}$ changing; in contrast, the P14 line is sensitive to both $\log g$ and $T_{\text {eff }}$. Only the grid of $T_{\text {eff }}=5250 \mathrm{~K}, \log g=2.0$, provides an acceptable match for the two Ca II lines, as well as the P14 line. Because the $\mathrm{S} / \mathrm{Ns}$ of the two Ca II lines are much higher than that of the P14 line, $\log g$ is better fitted than $T_{\text {eff. }}$. Therefore, the uncertainty of $\log g$ is estimated to be 0.3 , smaller than the grid step, but the uncertainty estimate of $T_{\text {eff }}$ is $250 \mathrm{~K}$, similar to the grid step. Through this approach, $T_{\text {eff }}$ is determined as $5250 \pm 250 \mathrm{~K}$ and $\log g$ as $2.0 \pm 0.3$.

\subsection{CO bandhead absorption}

The CO bandhead absorption longwards of $2.29 \mu \mathrm{m}$ is typical of late-type stars, whose outer atmospheric layers have the correct temperatures $(\sim 1000-3000 \mathrm{~K})$ to produce such features. The CO bandhead absorption generally start to appear in the spectra of late-G dwarfs, but their appearance could shift to earlier spectral types for supergiants (e.g., Wallace \& Hinkle 1997). However, low-mass Class II/III YSOs may also exhibit CO bandheads in absorption, because the effective temperature is not high enough to dissociate $\mathrm{CO}$ molecules, and the circumstellar veiling is less

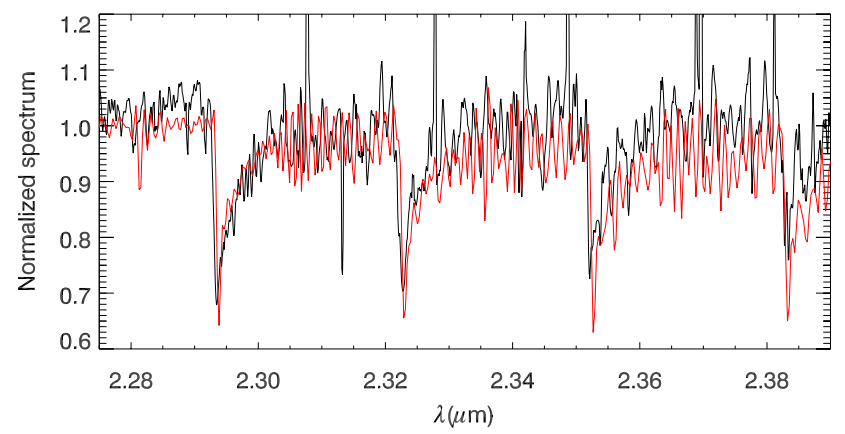

Fig. 5. Normalized X-shooter spectrum of CEN34 (black) in the wavelength range $2.28-2.39 \mu \mathrm{m}$, covering the CO bandhead absorption. For comparison, the reference spectrum of the mid-G supergiant HIP 107348 (SpT G5 Ib; Wallace \& Hinkle 1997) is shown in red. The spectrum of CEN 34 has been smoothed to the spectral resolution $(R \sim 3000)$ of the reference spectrum.

than that of Class I YSOs (Casali \& Eiroa 1996; Hoffmeister et al. 2006).

Figure 5 shows the CO bandhead absorption for CEN 34, as well as a reference mid-G supergiant. Since the CO bandhead absorption is strongly related to the $T_{\text {eff }}$ and to the surface gravity, González-Fernández \& Negueruela (2012, and reference therein) find a good correlation between the equivalent width of the $\mathrm{CO}$ bandhead in absorption (hereafter $\mathrm{CO}_{\mathrm{EW}}$ ) and spectral types for giants and supergiants: $\mathrm{CO}_{\mathrm{EW}}$ linearly increases with the decreasing $T_{\text {eff }}$, and is larger for supergiants than for giants of the same $T_{\text {eff }}$. Therefore the CO bandhead can be used to verify the above spectral type and luminosity class of CEN 34 . Following Fig. 2 in González-Fernández \& Negueruela (2012), the $\mathrm{CO}_{\mathrm{EW}} \approx 11$ A of CEN 34 suggests an early-K giant or mid-G supergiant, and the latter agrees well with the previously classified spectral type and luminosity class, if the involved uncertainty is considered.

Based on the spectral classification and synthetic spectral fitting presented above, CEN 34 has a spectral type of early-/mid-G $\left(T_{\text {eff }}=5250 \pm 250 \mathrm{~K}\right)$ and $\log g=2.0 \pm 0.3$, that is compatible with a supergiant. 


\subsection{Radial velocity relative to $L S R\left(V_{\mathrm{LSR}}\right)$}

Usually, newly formed stars conserve some kinematic imprint of their natal molecular cloud; i.e., stars having originated in the same cloud are expected to display similar radial velocities (Herbig 1977). Therefore, $V_{\mathrm{LSR}}$ measurements are an excellent tool for investigating the membership of stars in star-forming regions or stellar clusters.

In the case of M 17, comparison can be made to the young $\mathrm{O}$ - and B-type, whose membership is well established. For many of them, reliable spectral classification has been derived by Hoffmeister et al. (2008), primarily on the basis of lowresolution NIR spectroscopy with ISAAC complemented by higher resolution $(R=6600)$ optical-red GIRAFFE spectra covering the wavelength range 8200-9400 $\AA$. While the spectra of the B-type stars are featureless in the 8000-9000 $\AA$ range, accurate reference $V_{\mathrm{LSR}}$ values can be derived for 110 -type stars with strong ArI absorption lines at $8620 \AA$. In addition, the $\mathrm{X}$-shooter spectrum of the B7-type PMS star CEN 24/B 275 also serves as $V_{\text {LSR }}$ reference (Ochsendorf et al. 2011). Its Balmer series yield $V_{\mathrm{LSR}} \sim 24.7 \mathrm{~km} \mathrm{~s}^{-1}$ ). In total, we have calculated the $V_{\mathrm{LSR}}$ for 12 well-established OB-type members of M 17, and their mean $V_{\mathrm{LSR}}$ is $+29.4 \mathrm{~km} \mathrm{~s}^{-1}$, with a scatter of $7.1 \mathrm{~km} \mathrm{~s}^{-1}$. Furthermore, the prevalent $V_{\mathrm{LSR}}$ of the associated molecular clouds (M17-SW and M17-North) as obtained from radio and millimeter observations (Wilson et al. 2003; Xu et al. 2011) is $\sim+20 \mathrm{~km} \mathrm{~s}^{-1}$.

To determine the $V_{\mathrm{LSR}}$ of CEN 34, we use the Ca II triplet and the CO first overtone in the X-shooter spectrum. The lines of the triplet are fitted with Gaussian absorption profiles to derive their central wavelengths (see Table 1 ). Assuming $R=8800$, the velocity resolution of the X-shooter VIS arm is around $34 \mathrm{~km} \mathrm{~s}^{-1}$. Indeed, the central wavelengths fitted by Gaussian absorption profiles have errors below the velocity resolution. By testing different fitting parameters, the wavelength deviations are about $0.2 \AA$, corresponding to velocity resolution of $7 \mathrm{~km} \mathrm{~s}^{-1}$, i.e., $20 \%$ of the velocity resolution. Comparison to the rest wavelengths in air of the Ca II triplet (see Table 1) then yields a $V_{\mathrm{LSR}}$ of $-57 \pm 6 \mathrm{~km} \mathrm{~s}^{-1}$. To obtain an alternative estimate of its $V_{\mathrm{LSR}}$ from the CO first overtone, we cross-correlated CEN 34's spectrum with the high-resolution $(R=100000)$ vacuum rest-frame spectrum of Arcturus (see Fig. 6). For more details on this approach, the reader is referred to Figer et al. (2003). The spectrum of Arcturus was first resampled to match the lower spectral resolution $(R=5500)$ of the X-shooter NIR arm and then shifted in velocity by $-64 \mathrm{~km} \mathrm{~s}^{-1}$ to overlap with the spectrum of CEN 34 . The uncertainties involved in the velocity shift are dominated by the lower spectral resolution of the X-shooter spectrum and as before assumed to be on the order of $20 \%$ of the velocity resolution, or $11 \mathrm{~km} \mathrm{~s}^{-1}$. Obviously, the two independent $V_{\mathrm{LSR}}$ estimates for CEN 34 agree reasonably well within the errors. Thus, CEN 34 has a $V_{\text {LSR }}$ that is significantly distinct from those of the OB stars and from those of the associated molecular clouds. This suggests that CEN 34 is not a member of M 17, although one could argue that the high-mass stars in M 17 did conserve their original velocity, while stars with lower mass have been kicked out by tidal interactions.

The $V_{\mathrm{LSR}}$ of CEN 34 differs from that of any other molecular cloud within the inner Galaxy along the same line-ofsight $\left(l=15^{\circ}\right)$, by adopting the Galactic rotation model of Reid et al. (2009). A spiral arm lying beyond the Outer Arm in the first Galactic quadrant $\sim 23 \mathrm{kpc}$ from the Galactic center shows an LSR velocity of $-15 \mathrm{~km} \mathrm{~s}^{-1}$ at $l=15^{\circ}$

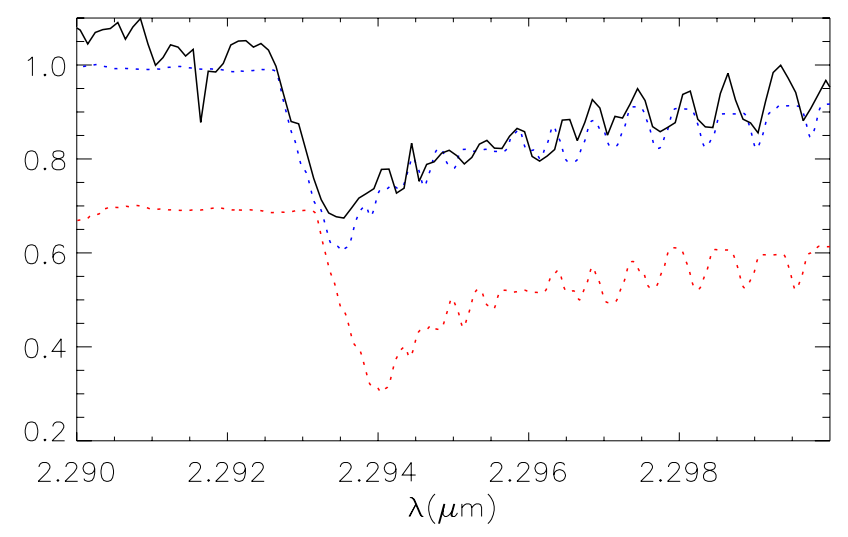

Fig. 6. Normalized CO first overtone bandhead of CEN 34 (black solid) and those of Arcturus (red and blue dotted lines). The red-dotted line presents the rest frame spectrum of Arcturus, while the blue-dotted one is the spectrum shifted by the velocity corresponding to the result from the cross correlation with respect to CEN 34's spectrum. The rest frame spectrum is shifted by arbitrary amounts along the vertical axis for presentation purposes.

Table 2. Multi-wavelength broadband photometry of CEN 34.

\begin{tabular}{lcccc}
\hline \hline Band & $\begin{array}{c}\lambda_{\mathrm{eff}} \\
\mu \mathrm{m}]\end{array}$ & Mag & $\begin{array}{c}\text { Flux } \\
(\mathrm{mJy})\end{array}$ & Ref. \\
\hline$B$ & 0.44 & $22.8 \pm 0.3$ & $3.13 \pm 0.86 \times 10^{-3}$ & 1 \\
$V$ & 0.55 & $18.82 \pm 0.05$ & $0.114 \pm 0.005$ & 1 \\
Johnson_R & 0.7 & $15.93 \pm 0.05$ & $1.25 \pm 0.06$ & 3 \\
DENIS_I & 0.79 & $14.482 \pm 0.04$ & $5.5 \pm 0.2$ & 2 \\
Johnson_I & 0.9 & $13.38 \pm 0.05$ & $12.0 \pm 0.5$ & 3 \\
2MASS_J & 1.23 & $10.402 \pm 0.024$ & $110.0 \pm 2.4$ & 4 \\
2MASS_H & 1.66 & $8.666 \pm 0.031$ & $349.9 \pm 9.9$ & 4 \\
2MASS_K & 2.16 & $7.788 \pm 0.023$ & $511.4 \pm 10.8$ & 4 \\
IRAC1 & 3.6 & $6.92 \pm 0.057$ & $479 \pm 25$ & 5 \\
L' & 3.78 & 6.77 & 495 & 1 \\
IRAC2 & 4.5 & $6.425 \pm 0.043$ & $483 \pm 19$ & 5 \\
IRAC3 & 5.8 & $5.834 \pm 0.034$ & $541 \pm 17$ & 5 \\
IRAC4 & 8.0 & $5.267 \pm 0.14$ & $493 \pm 64$ & 5 \\
$N$ & 10.5 & & $2500 \pm 730$ & 3 \\
$Q$ & 18.6 & & $5050 \pm 1395$ & 3 \\
\hline
\end{tabular}

References. (1) Hoffmeister (2008); (2) Epchtein et al. (1997); (3) Chini \& Krügel (1985); (4) Skrutskie et al. (2006); (5) Benjamin et al. (2003).

(Dame \& Thaddeus 2011). Therefore, it is not possible to estimate a kinematic distance for CEN 34 by its $V_{\mathrm{LSR}}$.

\subsection{Extinction and luminosity}

In Table 2, we collected the broadband photometric results for CEN 34 available from both the literature and the catalogs of recent infrared surveys (DENIS, 2MASS, and Spitzer GLIMPSE) to construct the SED of CEN 34 (see Fig. 7). The SED shows moderate infrared excess, most likely due to the presence of circumstellar material in an envelope and/or a disk. If CEN 34 is an evolved star the circumstellar envelope is a more plausible assumption than a circumstellar disk (Fujii et al. 2002).

We used the simulated annealing approach described in Liu et al. (2012) to model the observed SED and to estimate the mass of circumstellar material. We explored the scenario of a spherical envelope that is commonly adopted to mimic the dust shell surrounding a post-AGB star. The corresponding dust-density 


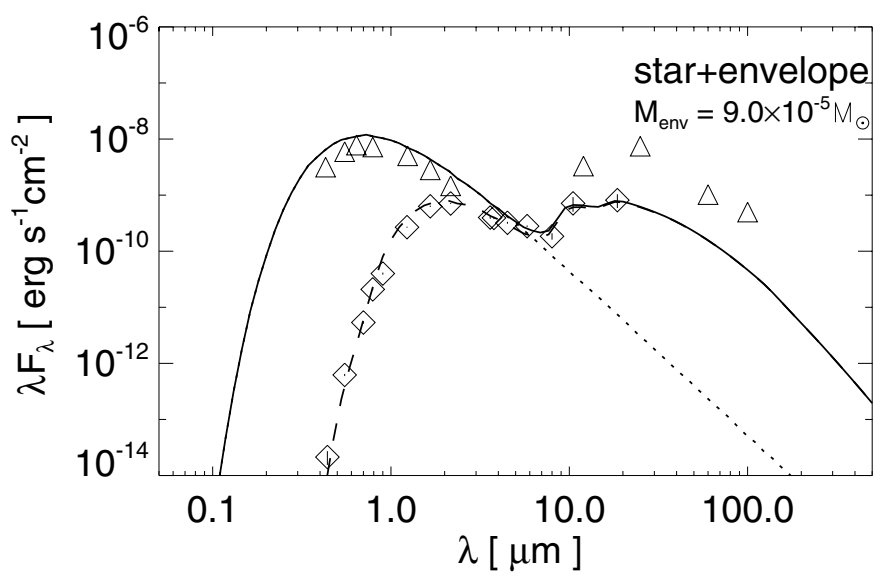

Fig. 7. Observed SED of CEN 34 generated from the photometric data listed in Table 2 (diamonds), together with the best-fitting model SED (dashed line) based on a star+envelope system of $M_{\text {env }}=9 \times 10^{-5} M_{\odot}$. After dereddening $\left(A_{\mathrm{v}}=11 \mathrm{mag}\right.$ ), the extinction free model SED (solid line) can be compared to the dereddened fluxes of the reference postAGB star IRAS 23304+6147 (triangles; Fujii et al. 2002). In addition, the best-fitting stellar blackbody $\left(T_{\text {eff }}=5260 \mathrm{~K}\right)$ is shown as a dotted line.

distribution is assumed to be a power law $\rho_{\mathrm{env}} \propto r^{-\alpha}$ with $\alpha=2$. Our best model fit is shown in Fig. 7. The observed SED at $\lambda \lesssim 4 \mu \mathrm{m}$, i.e. dominated by the stellar radiation, is reproduced by a star with $T_{\text {eff }}=5260 \mathrm{~K}$ reddened by $A_{V}=11 \mathrm{mag}$. The SED longward of $4 \mu \mathrm{m}$ can be reproduced by a spherical envelope of $9 \times 10^{-5} M_{\odot}$, yielding an optical depth $\tau_{v}=0.29$ for the circumstellar material along the line-of-sight. This corresponds to $A_{V}=0.3 \mathrm{mag}$ and is only a small fraction of the total extinction of $A_{V}=11 \mathrm{mag}$ mentioned above. Considering that Hoffmeister et al. (2008) derived a foreground extinction of 2 mag toward M 17, the largest fraction of extinction originates inside the $\mathrm{H}$ II region and behind it, in case CEN 34 is not a cluster member. Therefore, the distance of CEN 34 is at least greater than $2 \mathrm{kpc}$, the distance of M 17. With this lower limit of CEN 34's distance, the lower limit of the luminosity is determined as $1600 L_{\odot}$. On the other hand, the derived temperature and surface gravity also constrain the absolute magnitude of CEN 34. Using the calibration from (Straizys \& Kuriliene 1981) we obtain $M_{V}=-4 \mathrm{mag}$, which transforms into a photometric distance of $2.3 \mathrm{kpc}$. However, this distance estimate has a large error due to the uncertainty of $\log g$.

\section{Discussion}

In Fig. 8 we outline the location of CEN 34 in the HertzsprungRussell diagram (HRD) based on effective temperature and luminosity ranges constrained in Sects. 3.2 and 3.5. Comparison is made to two distinct sets of theoretical evolutionary tracks and isochrones: while in the lefthand panel, we compare to high-mass star formation models of Hosokawa et al. (2010), in the righthand panel we overplot post-main sequence isochrones of Marigo et al. (2008). Obviously both high-mass YSOs and evolved stars are compatible with the observations of CEN 34.

As demonstrated by the numerical simulations of Hosokawa et al. (2010), very young and accreting high-mass YSOs tend to have rather low $T_{\text {eff }}$ and large radius, similar to those obtained for CEN 34 in Sect. 3.2. However, if we assume that CEN 34 is associated with the M 17 region and located at a distance of $2 \mathrm{kpc}$, its $T_{\text {eff }} \approx 5250 \mathrm{~K}$ and luminosity $L_{*} \gtrsim 1600 L_{\odot}$ place CEN 34 in an area of the HRD where the numerical models suggest accretion rates in excess of $4 \times 10^{-3} M_{\odot} / y r$. Nevertheless, accretion rates may vary strongly with the evolution of high-mass YSOs. As a consequence, CEN 34 does not need to maintain such a high accretion rate throughout its entire formation process. Such extremely high accretion rates are usually expected to correlate with the presence of a very massive $\left(\sim 1 M_{\odot}\right)$ envelope/disk surrounding the central high-mass YSO, which obviously contradicts to the amount of circumstellar material actually estimated for CEN $34\left(\sim 10^{-4} M_{\odot}\right.$; see Sect. 3.5). This discrepancy could be circumvented by a scenario in which the high-mass YSO has already accumulated most of its circumstellar material in a very short time span, so that the circumstellar material becomes optically thin. We speculate that CEN 34 might be in a phase of gravitational contraction, during which it will not gain more material from its surroundings and will move to the left in the HRD.

To solve the $V_{\mathrm{LSR}}$ inconsistency between CEN 34 and M 17, one might argue that CEN 34 has been ejected from M 17. Recently, Gvaramadze et al. (2013) have reported that even O-type stars can be ejected from a massive cluster because of a dynamical few-body encounter in the cluster's core. If we propose that CEN 34 is dynamically kicked o the cluster center, then the time that CEN 34 needs to move to the current position relative to the cluster center can be used to test our speculation. The distance from CEN 34 to the cluster center is about $1 \mathrm{pc}$, which is the relative distance projected to the sky plane. Assuming $1 \mathrm{pc}$ to the relative distance perpendicular to the sky plane, the $V_{\mathrm{LSR}}$ difference of $\sim 80 \mathrm{~km} \mathrm{~s}^{-1}$ yields a time of $\sim 1.2 \times 10^{4}$ yrs, which is needed for CEN 34 to move to the current position. This traveling time is comparable to the age of CEN 34 if it had accumulated the bulk of its mass with accretion rate $>4 \times 10^{-3} M_{\odot} / \mathrm{yr}$ within a period of $\sim 10^{3}$ yrs. A byproduct of this ejection scenario is to place CEN 34 in an isolated environment where feeding through its parental cloud cannot be continued.

Alternatively, besides the fact that the spectrum of CEN 34 shows very similar characteristics to those of post-AGB stars, which typically are late-type supergiants with spectral absorption features as discussed in Sects. 3.1-3.3 (Szczerba et al. 2007), the overall shape of CEN 34's SED is reminiscent of post-AGB stars. To foster the hypothesis of CEN 34 being a post-AGB star, we compared its dereddened SED (Fig. 7) to that of a reference post-AGB star of spectral type G2 Ia (IRAS 23304+6147) from Fujii et al. (2002), located at a distance $(d \sim 4.5 \mathrm{kpc})$. IRAS $23304+6147$ has mid-infrared excess that is slightly stronger than that of CEN 34, most likely due to larger amount of circumstellar material. In view of the similar spectral types and luminosity classes of CEN 34 and IRAS 23304+6147, their comparable optical and NIR fluxes imply a comparable distance, too. Because the optical and NIR fluxes of CEN 34 are still somewhat higher than those of IRAS $23304+6147$, the distance of IRAS $23304+6147$ (i.e., $4.5 \mathrm{kpc}$ ) serves as upper limit for CEN 34's distance. Considering the most likely range of CEN 34's distance ( $2 \mathrm{kpc} \lesssim d \lesssim 4.5 \mathrm{kpc}$ ), the corresponding luminosity range is easily derived $\left(1600 L_{\odot} \lesssim L_{*} \lesssim 8100 L_{\odot}\right)$. By comparing this luminosity range and the effective temperature to post-main sequence isochrones of Marigo et al. (2008) (see the right panel of Fig. 8), we constrain the ranges of stellar mass and age to 5-7 $M_{\odot}$ and $100-50 \mathrm{Myr}$, respectively. This would suggest that CEN 34 is an evolved intermediatemass star. While post-AGB stars typically yield prominent farinfrared emissions (e.g., Suárez et al. 2006; Szczerba et al. 2007), far-infrared flux measurements of CEN 34 (e.g., from IRAS or AKARI) are highly contaminated by the strong dust 


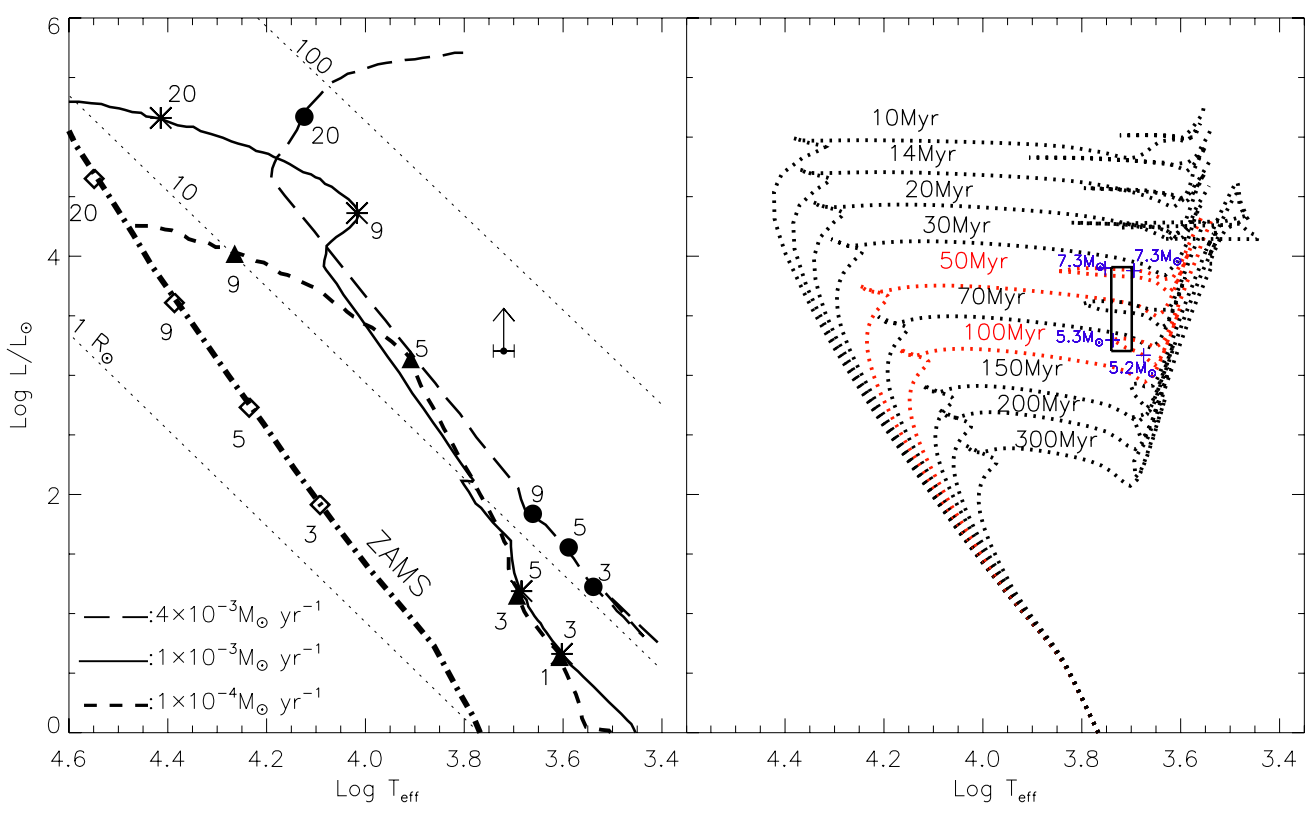

Fig. 8. Position of CEN 34 in the Hertzsprung-Russell diagram (HRD) with a well determined effective temperature (5250 $\pm 250 \mathrm{~K})$ and loosely constrained luminosity $\left(1600 L_{\odot} \lesssim L_{*} \lesssim 8100 L_{\odot}\right)$. Left panel: the position of CEN 34 denoted by the full dot with error bar is compared with the model of massive star formation via disk accretion (Hosokawa et al. 2010). The luminosity of $1600 L_{\odot}$ adopted here is the lower limit (denoted by the upward arrow) when the distance of CEN 34 is assumed to be $2 \mathrm{kpc}$. Right panel: the position of CEN 34 (the rectangle representing the uncertainties of $T_{\text {eff }}$ and $L_{*}$ ) is placed against the isochrones of post-main-sequence evolution (Marigo et al. 2008). The lower and upper isochrones (roughly the $100 \mathrm{Myr}$ and $50 \mathrm{Myr}$ ones, respectively) that enclose the position of CEN 34 are highlighted in red, and the corresponding masses are highlighted in blue.

continuum of the M17 S-bar. For this reason, the $60 \mu \mathrm{m}$ and $100 \mu \mathrm{m}$ fluxes listed for CEN 34 in the IRAS Point Source Reject Catalog (released in 1989) are unreliable, because of the large IRAS beam size $(\sim 1$ arcmin at $60 \mu \mathrm{m})$; although AKARI observed with higher spatial resolution and better sensitivities than IRAS, unfortunately the AKARI/FIS All-Sky Survey Point Source Catalogue (Yamamura et al. 2010) does not return an entry matching the position of CEN 34 .

\section{Summary and conclusions}

Originally considered a high-mass YSO candidate in the M 17 star-forming region, CEN 34 has been reclassified as an early-/mid-G supergiant on the basis of the new X-shooter spectrum. The SED of CEN 34 resembles that of either a high-mass YSO or an evolved intermediate-mass star (likely a post-AGB star) with a visual extinction $A_{V}=11 \mathrm{mag}$ that it is dominated by interstellar extinction. If CEN 34 is a high-mass YSO, its derive properties $\left(T_{\text {eff }}, \log g\right.$, and $\left.V_{\mathrm{LSR}}\right)$ can be explained by a scenario where the star has been ejected from the M 17 cluster. In this case CEN 34 had accumulated the bulk of its mass during the first $\sim 10^{3}$ years, and it is currently gravitationally contracting with only little circumstellar material left. If, on the other hand, CEN 34 is an evolved star of intermediate mass, its luminosity is in the range $1600 L_{\odot} \lesssim L_{*} \lesssim 8100 L_{\odot}$, corresponding to a distance range of $2 \mathrm{kpc} \lesssim d \lesssim 4.5 \mathrm{kpc}$. Comparison to the isochrones of post-main sequence evolution then suggests that CEN 34 has a mass of 5-7 $M_{\odot}$ and an age of 50-100 Myr, which are reminiscent of a post-AGB star.

In conclusion, the ambiguities concerning infrared excess and spectral types between high-mass YSOs and evolved intermediate-mass stars prevent a unique classification of CEN 34. The arguments for interpreting CEN 34 as a high-mass YSO are not as straightforward as in the case of a post-AGB star, and they are based on a number of assumptions. For the time being, we prefer the interpretation of a post-AGB star and hope that a future, more accurate distance estimate will settle the issue.

Acknowledgements. We acknowledge the anonymous referee whose comments helped to clarify the paper at several points. The ESO Paranal staff is acknowledged for obtaining the X-shooter spectrum of CEN 34 in Service Mode. M.F. acknowledges the support by the NSFC through grants 11203081 . This publication used data products from the Two Micron All Sky Survey, which is a joint project of the University of Massachusetts and the Infrared Processing and Analysis Center/California Institute of Technology, funded by the National Aeronautics and Space Administration and the National Science Foundation. This research made use of the SIMBAD database, operated at the CDS, Strasbourg, France. This research made use of the NASA/IPAC Infrared Science Archive (IRSA) which is operated by the Jet Propulsion Laboratory, California Institute of Technology, under contract with the National Aeronautics and Space Administration. This research made use of NASA's Astrophysics Data System Bibliographic Services.

\section{References}

Andrillat, Y., Jaschek, C., \& Jaschek, M. 1995, A\&AS, 112, 475

Benjamin, R. A., Churchwell, E., Babler, B. L., et al. 2003, PASP, 115, 953

Carquillat, M. J., Jaschek, C., Jaschek, M., \& Ginestet, N. 1997, A\&AS, 123, 5

Casali, M. M., \& Eiroa, C. 1996, A\&A, 306, 427

Chen, Z., Jiang, Z., Wang, Y., et al. 2012, PASJ, 64, 110

Chini, R., \& Krügel, E. 1985, A\&A, 146, 175

Chini, R., Hoffmeister, V., Kimeswenger, S., et al. 2004, Nature, 429, 155

Dame, T. M., \& Thaddeus, P. 2011, ApJ, 734, L24

De Medeiros, J. R., Udry, S., Burki, G., \& Mayor, M. 2002, A\&A, 395, 97 D’Odorico, S., Dekker, H., Mazzoleni, R., et al. 2006, in SPIE Conf. Ser., 6269 Epchtein, N., de Batz, B., Capoani, L., et al. 1997, The Messenger, 87, 27

Figer, D. F., Gilmore, D., Kim, S. S., et al. 2003, ApJ, 599, 1139

Fujii, T., Nakada, Y., \& Parthasarathy, M. 2002, A\&A, 385, 884

Goldoni, P. 2011, Astron. Nachr., 332, 227

González-Fernández, C., \& Negueruela, I. 2012, A\&A, 539, A100

Gray, R. O., \& Corbally, J. C. 2009, Stellar Spectral Classification (Princeton University Press) 
Gvaramadze, V. V., Kniazev, A. Y., Chené, A.-N., \& Schnurr, O. 2013, MNRAS, 430, L20

Hanson, M. M., Howarth, I. D., \& Conti, P. S. 1997, ApJ, 489, 698 Herbig, G. H. 1977, ApJ, 214, 747

Hoffmeister, V. H. 2008, Ph.D. Thesis, Ruhr-Universität Bochum Hoffmeister, V. H., Chini, R., Scheyda, C. M., et al. 2006, A\&A, 457, L29

Hoffmeister, V. H., Chini, R., Scheyda, C. M., et al. 2008, ApJ, 686, 310

Hosokawa, T., Yorke, H. W., \& Omukai, K. 2010, ApJ, 721, 478

Jiang, Z., Yao, Y., Yang, J., et al. 2002, ApJ, 577, 245

Liu, Y., Madlener, D., Wolf, S., Wang, H., \& Ruge, J. P. 2012, A\&A, 546, A7

Marigo, P., Girardi, L., Bressan, A., et al. 2008, A\&A, 482, 883

Munari, U., \& Tomasella, L. 1999, A\&AS, 137, 521

Munari, U., Sordo, R., Castelli, F., \& Zwitter, T. 2005, A\&A, 442, 1127

Nielbock, M., Chini, R., Jütte, M., \& Manthey, E. 2001, A\&A, 377, 273

Nielbock, M., Chini, R., Hoffmeister, V. H., et al. 2007, ApJ, 656, L81

Nürnberger, D. E. A., Chini, R., Eisenhauer, F., et al. 2007, A\&A, 465, 931

Ochsendorf, B. B., Ellerbroek, L. E., Chini, R., et al. 2011, A\&A, 536, L1

Povich, M. S., Churchwell, E., Bieging, J. H., et al. 2009, ApJ, 696, 1278
Reader, J., Corliss, C. H., Wiese, W. L., \& Martin, G. A. 1980, Wavelengths and transition probabilities for atoms and atomic ions: Part 1. Wavelengths, part 2. Transition probabilities

Reid, M. J., Menten, K. M., Zheng, X. W., et al. 2009, ApJ, 700, 137

Skrutskie, M. F., Cutri, R. M., Stiening, R., et al. 2006, AJ, 131, 1163

Straizys, V., \& Kuriliene, G. 1981, Ap\&SS, 80, 353

Suárez, O., García-Lario, P., Manchado, A., et al. 2006, A\&A, 458, 173

Szczerba, R., Siódmiak, N., Stasińska, G., \& Borkowski, J. 2007, A\&A, 469, 799

Valdes, F., Gupta, R., Rose, J. A., Singh, H. P., \& Bell, D. J. 2004, ApJS, 152, 251

Vernet, J., Dekker, H., D’Odorico, S., et al. 2011, A\&A, 536, A105

Wallace, L., \& Hinkle, K. 1997, ApJS, 111, 445

Wilson, T. L., Hanson, M. M., \& Muders, D. 2003, ApJ, 590, 895

Xu, Y., Moscadelli, L., Reid, M. J., et al. 2011, ApJ, 733, 25

Yamamura, I., Makiuti, S., Ikeda, N., et al. 2010, VizieR Online Data Catalog: II/298

Zinnecker, H., \& Yorke, H. W. 2007, ARA\&A, 45, 481 